\title{
UN ESTUDIO EXPLORATORIO DE FORMACIÓN EN STORYTELLING Y SUS CLAVES, PARA SU APLICACIÓN EN LA EDUCACIÓN FOR- MAL DE ADULTOS
}

\author{
EXPLORATORY STUDY OF NARRATIVE TRAINING AND ITS KEYS, \\ FOR ITS APPLICATION IN FORMAL ADULT EDUCATION \\ UNE ÉTUDE EXPLORATOIRE DE LA FORMATION À LA NARRATION ET DE SES \\ CLÉS, POUR SON APPLICATION DANS L'ÉDUCATION FORMELLE DES ADULTES \\ José Luis Galar Gimeno \\ Universidad de Zaragoza
}

\begin{abstract}
Resumen:
Este estudio tiene el propósito de averiguar si el uso de , muy utilizado en disciplinas que buscan comunicar eficazmente (marketing, publicidad, política o derecho procesal), puede resultar eficaz en el ámbito de la educación formal de adultos. Para dar respuesta a la pregunta de investigación anterior se fijan dos objetivos: 1) conocer si el storytelling es entrenable como técnica; 2) indagar acerca de cuáles son sus claves. Para ello se plantea un análisis exploratorio a partir de la experiencia de informantes expertos, profesionales que utilizan el storytelling en el ejercicio de sus profesiones, y, además, imparten docencia formal de adultos transfiriendo su experticia en el uso del storytelling desde su ámbito profesional al aula. Los resultados de la investigación ponen de manifiesto que el storytelling puede ser una técnica entrenable, que las claves para un storytelling eficaz residen en potenciar las habilidades interpersonales del comunicador desde el profundo autoconocimiento, en un buen relato que implique el componente emocional del alumnado, y que el relato es indisociable del comunicador para conseguir una voz personal.
\end{abstract}

Palabras clave: , estrategias comunicativas, discurso persuasivo, retórica, competencias comunicativas. 


\begin{abstract}
This study aims to find out if the use of storytelling, widely used in disciplines that seek to communicate effectively (marketing, advertising, politics or procedural law), can be effective in the field of formal adult education. To answer the previous research question, two objectives are set: 1) to know if storyteIling is trainable as a technique; 2) inquire about what their keys are. For this, an exploratory analysis is proposed based on the experience of expert informants, professionals who habitually use storytelling in the exercise of their professions, and, in addition, provide formal adult teaching transferring their expertise in the use of storytelling from their professional field. To the classroom. The results of the research show that storytelling can be a trainable technique, that the keys to effective storytelling lie in enhancing the communicator's interpersonal skills from deep self-knowledge, in a good story that involves the emotional component of the students, and that the story is inseparable from the communicator in the achievement of a personal, differential and irreplaceable voice.
\end{abstract}

Key words: storytelling, communication strategies, persuasive discourse, rhetoric, communication skills.

\title{
Résumé
}

Cette étude vise à savoir si l'utilisation de la narration, largement utilisée dans les disciplines qui cherchent à communiquer efficacement (marketing, publicité, politique ou droit procédural), peut être efficace dans le domaine de l'éducation formelle des adultes. Pour répondre à la question de recherche précédente, deux objectifs sont fixés : 1) savoir si le storytelling est entraînable en tant que technique ;2) se renseigner sur ses clés. Pour cela, une analyse exploratoire est proposée sur la base de l'expérience d'informateurs experts, professionnels qui utilisent habituellement la narration dans l'exercice de leurs professions, et, en plus, dispensent un enseignement formel pour adultes transférant leur expertise dans l'utilisation de la narration de leur domaine professionnel à la salle de classe. Les résultats de la recherche montrent que la narration peut être une technique entraînable, que les clés d'une narration efficace résident dans l'amélioration des compétences interpersonnelles du communicateur à partir d'une profonde connaissance de soi, dans une bonne histoire qui implique la composante émotionneIle des élèves, et que l'histoire est indissociable du communicateur dans la réalisation d'une voix personnelle, différentielle et irremplaçable.

Mots-clés: storytelling, stratégies de communication, discours persuasif, rhétorique, compétences en communication. 


\section{INTRODUCCIÓN}

En este estudio se entenderá el storytelling como el conjunto de actividades, procesos y hechos formativos formales e informales orientados a la creación de historias a través de varios instrumentos y técnicas que contribuyen a mejorar la calidad del aprendizaje, y presenta una doble naturaleza, por una parte, se trata de un método útil en la práctica del docente y a la vez resulta una herramienta útil para la acción didáctica (Paiano, 2019).

La revisión de la bibliografía indica que el uso del relato o storytelling ha sido adoptado por numerosos colectivos donde la comunicación persuasiva resulta necesaria como el marketing, la publicidad, el derecho procesal, la política o la religión (Álvarez, 2014; Bautista, 2009; Harari, 2016; López y Vargas, 2013; Salmon, 2008). Se ha incorporado como técnica comunicativa novedosa y emergente en muchas disciplinas y países, pero no ha suscitado un entusiasmo de paralela intensidad en el campo científico académico español (Vázquez, 2016). Tampoco lo ha incorporado la comunidad educativa española que suele abordar su comunicación más como un proceso de transmisión de contenidos que con la capacidad de conectar con las emociones, las cuales están relacionadas con la cognición según Duveskog et al. (2012) y Tolisano (2009). Sin embargo, autores como Porter (2008) ven en el storyteIling una técnica que puede contribuir a "guiar", "motivar", "inspirar" y "educar" debido a su capacidad de conectar cognición y emoción. En algunos artículos recientes se sostiene que la focalización en lo cognitivo es insuficiente, y que la comprensión ha de ir acompañada de la activación de una respuesta emocional, no siendo potente el mensaje comprendido, sino el que moviliza en la dirección adecuada (Ferrés y Masanet, 2017; Mora, 2017).

En este sentido, Mora (2017) sostiene que un buen docente debe ser capaz de captar la atención de los alumnos, porque sin atención no hay aprendizaje. Para este autor, esa capacidad nace de la habilidad del profesor para convertir la clase en un cuento, una historia, creando una envoltura curiosa, atractiva, cualquiera que sea la temática de la que se trate. Una historia con un principio que ilumine al tiempo 
que provoque, un desarrollo que interese (introduciendo novedad, sorpresa, complejidad), y un final que resuma lo dicho y despierte el interés por la próxima clase. Es decir, perseguir la activación emocional a través del relato construido ad hoc (narratividad), algo que conecta con lo sostenido ya en Goleman (2006): involucrar las emociones permite transformar y mejorar el pensamiento del alumno al dirigir su atención hacia contenidos relevantes y significativos.

Teniendo en cuenta lo anterior podría tener sentido una formación en el uso del storytelling dirigida a docentes, pues la importancia del uso del relato o storytelling reside en que es algo connatural al ser humano (Paiano, 2019). No se trata de algo nuevo, pues las historias son tan antiguas como la gente y muy importantes en el progreso humano (Porter, 2008). Los seres humanos expresamos nuestras creencias, deseos y esperanzas con historias en un intento de explicarnos y comprender a los demás (Greene, 1996). Crear sentido es algo central en el funcionamiento psicológico humano (Bietti et al., 2019), por este motivo el relato ha estado presente en todas las épocas, lugares y sociedades bajo sus infinitas formas, empezó con la historia misma de la Humanidad y no ha habido ni hay un pueblo sin relato (Barthes et al., 1970).

Contar historias fue una de las primeras estrategias de comunicación utilizadas por la humanidad y todavía se usa en una variedad de culturas para transmitir tradiciones, costumbres y recuerdos (Cleverley-Thompson, 2018). Sostiene Harari (2016) que "los relatos hacen las veces de cimientos y pilares de las sociedades humanas." (p. 202). Sugiyama (2001) incluso llega a proponer que el storytelling es una función adaptativa del ser humano. Lo argumenta basándose en su universalidad, ya que emergió de forma independiente en diferentes lugares de la Tierra, incluso en pueblos aislados por completo del resto, y se desarrolló de forma próspera en la ontogenia de la civilización. Bietti et al. (2019) argumentan que el storytelling representa un elemento clave en la creación y propagación de la cultura, y que las cuestiones que hacen de él una función adaptativa son la posibilidad de: a) persuadir (motivar) la audiencia en algún sentido, b) transmitir información relevante para la 
supervivencia evitando el alto coste de adquirir de primera mano de esa información, y c) mantener la cohesión grupal para cumplir obligaciones o cooperar a nivel colectivo.

En busca de esa evidencia que supone al storytelling una función adaptativa, Bietti et al. (2019) se basan en tres fuentes: la primera de Stubbersfield et al. (2017) que se orienta sobre la estructura y el contenido de la narrativa enfocando a varios aspectos del sistema cognitivo humano; la segunda es de Mellmann (2012) quien acude a la motivación instintiva de los participantes para engancharse a la actividad del storytelling; y la tercera de Mar (2011) que aduce la existencia de circuitos neurales especializados en producir y comprender historias y relatos. Lo recogido en estas tres fuentes conecta con los nuevos descubrimientos y progresos en neuroeducación basada en la neurociencia, que reconoce la emoción como la puerta de entrada al aprendizaje (Mora, 2017).

Esta investigación pretende explorar desde la experticia de los participantes qué claves hacen del storytelling una técnica eficaz para mejorar el proceso de aprendizaje del alumnado conectando con la parte subjetiva de su emoción (Galar et al., 2021).

\section{MÉTODO}

Para dar respuesta a los objetivos fijados (conocer si el storytelling es entrenable como técnica e indagar acerca sus claves) se opta por la metodología cualitativa, planteando una investigación exploratoria (Hernández et al., 2010; Merriam y Tisdell, 2009; Morales, 2015; Yin, 2015) con técnica de análisis Delphi, un método fuertemente consolidado en la investigación educativa (Patton, 1987), adaptable a las necesidades de cada momento (Báez, 2006; Pozo et al., 2007; Reguant y Torrado, 2016; Webler et al., 1991). Acudiendo a un juicio intersubjetivo, se pretende reducir al máximo los sesgos y limitaciones que podrían presentar las opiniones de un solo experto lo que aumenta la fiabilidad de los resultados. (Báez, 2006; Cabero y Infante, 2014; Reguant y Torrado, 2016). 


\section{Participantes}

El panel de expertos se elige de forma no probabilística e intencional, una muestra representativa en base a lo que el estudio pretende perfilar desde la pregunta de investigación y los objetivos fijados (Flick, 2012). Lo forman 12 expertos en el uso profesional del storytelling que además imparten docencia en el ámbito educativo formal de adultos y que transfieren de forma consciente su experticia con el storytelling al aula. Todos ellos, en algún momento de su carrera profesional, han formado parte del cuerpo docente universitario como profesores asociados en universidades tanto públicas como privadas. La media de edad se sitúa en los 45 años, siendo 29 el valor mínimo y 65 años el valor máximo. En cuanto al sexo el 66`6\% son hombres y el 33’4 mujeres. En cuanto a su formación, 3 ingenieros (1 doctor en Políticas Públicas, 1 máster en Ingeniería y Diseño Industrial, 1 diplomado en Turismo); 5 licenciados en Derecho (1 doctor en Sociología, 1 máster IESE); 1 licenciado en Sociología (doctor en Sociología); 1 licenciado en Bioquímica y Veterinaria (doctor en Ciencias); 1 licenciado en Veterinaria (doctor en Marketing); 1 licenciado en Teología y en Filosofía e Historia. Todos han cursado algún tipo de formación sobre uso del storytelling como técnica de comunicación persuasiva.

\section{Procedimiento}

Delimitado el problema se planteó la pregunta de investigación, se fijaron los objetivos, se llevó a cabo una revisión de la bibliografía, y se compuso el marco teórico del estudio. A partir de ahí emergieron los siguientes núcleos de interés: a) habilidades de un comunicador (docente); b) qué valora la audiencia (alumnado) en la comunicación docente-discente; c) si el uso del storytelling hace el mensaje más comprensible; d) qué consigue implicar el componente emocional de la audiencia; e) diferencia entre persuasión y manipulación; f) relación entre factor humano y las tecnologías de la información y comunicación (TIC); g) entrenabilidad en la técnica del storytelling vía formación.

Para recoger la máxima información sobre los núcleos de interés, el instru- 
mento por el que se optó fue la entrevista semiestructurada debido a la especial relevancia que cobra en esta investigación el punto de vista de los entrevistados (Hernández, 2014). Desde los núcleos de interés se construyó un protocolo de 27 tópicos. El protocolo se validó enviándolo por correo electrónico a tres expertos: un director de grado en periodismo, una profesora de investigación de mercados, marketing promocional e introducción al fundraising, y una periodista del gabinete de comunicación de presidencia de comunidad autónoma. Los expertos detectaron que algunos de los ítems incluidos en el protocolo estaban planteados de forma que podían inducir a los entrevistados a una respuesta positiva. Se revisó la redacción de los ítems indicados por los expertos y se volvieron a redactar siguiendo sus indicaciones para evitar el sesgo de expectancia. Se volvió a enviar el protocolo a los expertos una vez modificado, siendo validado por unanimidad "por su adecuación a lo que se pretende investigar".

Con el protocolo validado se llevó a cabo la entrevista semiestructurada con cada uno de los expertos de forma individual y en días diferentes, en el mismo espacio físico. Se les pidió autorización para grabar la entrevista mientras se les informaba de que la duración sería de aproximadamente 60 minutos. Realizadas las 12 entrevistas se transcribió fielmente cada una de ellas utilizando un documento Word. El investigador sometió los textos resultantes a múltiples lecturas en constante reflexividad e iteratividad (Sandín, 2003), surgiendo del proceso un listado de categorías vinculadas a los núcleos de interés. Se remitió el listado de categorías a los participantes en busca del acuerdo sobre la categorización. El resultado fue el acuerdo por unanimidad sobre el listado propuesto y la emergencia de dos nuevas categorías (contexto y reputación), que se incluyeron en el listado a posteriori (Díaz et al., 2013).

El análisis se realizó utilizando el paquete informático Nvivo 11 Plus. Se trabajó con las opciones del "mapa jerárquico", la "nube de palabras" y el "árbol de palabras" para buscar las clases estadísticas más cercanas en cuanto los objetivos fijados en la investigación. Finalmente, para realizar la necesaria reducción de categorías que 
conviene a un estudio exploratorio (Morales, 2015; Villardón, 2007) se recurrió al dendograma o análisis de conglomerados. Técnica descriptiva, ateórica y no inferencial que busca agregar variables tratando de lograr la máxima homogeneidad en cada grupo y la mayor de diferencia entre grupos mediante mediciones de similitud del coeficiente de correlación de Pearson (De La Fuente, 2011).

El producto del análisis de las entrevistas a los expertos produjo una sencilla respuesta estadística. Se subraya que la sencillez no le resta rigor, pues en opinión de Reguant y Torrado (2016) "debido al número reducido de participantes (entre 6 y 30), el método no está destinado a producir resultados significativamente estadísticos." (p. 91).

\section{RESULTADOS}

Las categorías vinculadas a los núcleos de interés que surgen tras el análisis de las transcripciones de las entrevistas a los expertos quedan recogidas en la tabla 1.

\section{Tabla 1.}

Categorías surgidas respecto a los núcleos de interés y sus definiciones

\begin{tabular}{ll} 
Categorías & Definición \\
\hline Comunicador (docente) & Habilidades del comunicador. \\
Contenido eficaz & Elementos del relato. \\
Contexto & Su influencia en el proceso de la comunicación. \\
Escenificación & Puesta en escena. \\
Formación & Entrenabilidad vía formación \\
Lenguaje & Lenguaje apreciado por la audiencia. \\
Limitado & Ámbitos en los que no se considera útil el storytelling. \\
Motivo & Por qué utilizar el storytelling. \\
Palancas emocionales & Elementos que implican el componente subjetivo de la \\
emoción en la audiencia
\end{tabular}


Sorpresa

TIC (Tecnologías de la Información y Comunicación)

Retribución
Emoción inicial para despertar el interés y mantener la atención de la audiencia.

Complementariedad factor humano (orador/docente) y TIC

Lo que la audiencia (alumnado) espera recibir al prestar atención al orador (docente)

Respecto al primer objetivo (conocer si el storytelling es entrenable como técnica) el análisis de conglomerados mediante mediciones de similitud del coeficiente de correlación de Pearson, descrito en apartado dedicado al procedimiento, y recogido en la figura 1, indica que el storytelling como técnica es considerado por el panel de expertos como una habilidad entrenable vía formación, y, además, según esta agrupación, tanto la formación (entrenabilidad) como la persuasión (entendida como narrativa motivacional) pueden fusionarse dentro de la misma categoría. A continuación, las respuestas más representativas de los resultados respecto al primer objetivo':

- "No debe ser un curso solo de storytelling, sino que en un curso de comunicación incluyan las herramientas de storytelling. Incluso serían interesantes unas clases de teatro."

- "El storytelling es una técnica y como la técnica de escritura o hablar en público es enseñable, entrenable, evaluable."

- "Por supuesto, estoy plenamente convencida que debemos aprender a comunicar con historias."

- "Si haces un curso puedes aprender un modelo, pero además del modelo puede haber otras cosas, como uso del lenguaje, recursos teatrales... Modular el comportamiento según el mensaje siendo creíble, necesita herramientas que debes aprender a manejar."

$1 \mathrm{El}$ entrecomillado recoge expresiones extraídas de las transcripciones de las entrevistas realizadas a los participantes, que resultan representativas de las respuestas unánimes dadas por los doce expertos. 
- "Es muy importante dar herramientas a los docentes, más que herramientas ideas, una especie de hipótesis contrastadas y ciertas de forma que digan si usted hace esto, esto, esto y esto, usted va a conseguir llegar mejor a sus objetivos. Y por ejemplo es, si usted cuenta historias, usted va a llegar mejor a los objetivos, si usted tiene una buena puesta en escena gana en emoción."

\section{Figura 1.}

Clúster que muestra la relación entre las categorías coincidentes con el primer objetivo.

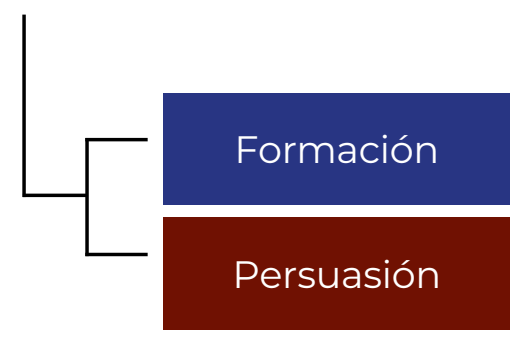

Respecto al segundo objetivo (indagar acerca de cuáles son las claves de la técnica del storytelling), el análisis de conglomerados recogido en la figura 2, indica que las claves están en²:

1. Palancas emocionales,

. "Un relato con el que la audiencia se sienta identificada."

. "Un relato que conecta con valores éticos, superación personal y motivación."

2. En las habilidades del comunicador

- "Capacidad de utilizar un lenguaje sencillo, fácil, comprensible, directo, no rebuscado."

- "Ser consciente de la reacción emocional de la audiencia para ir adaptando su tono, su rapidez en función de las emociones que va consiguiendo".

- "Ser un buen comunicador exige una autodisciplina, hasta que las cosas te salgan casi sin pensarlo."

$2 \quad$ Igual que en nota anterior. 
- "Conocimiento técnico y atención psicológica, que, al final, es un ejercicio de empatía con tus interlocutores, conocer a aquellos a quienes te vas a dirigir, y en función de esto vas desarrollando las restantes, es una conjunción de finalidad y medio."

3. Retribución

- "Si no se llevan nada enseguida desconectan".

- "Cada diez minutos hay que sembrar en el auditorio una semilla, dejar una frase. Que le dejemos algún mensaje que los lleve a un estado de reflexionar, que en este tiempo ya es suficiente. Si quisiéramos ser más ambiciosos, no solo reflexionar sino actuar, llamar a la acción. En este tiempo de tantas conferencias, formación, de tantos medios disponibles para formarse, simplemente el hecho de reflexionar ya es un punto importante. Si la gente actuara, pues todavía mucho mejor. Pero los inputs pueden llegar a ser tantos que solo el hecho de la reflexión ya es importante."

- "Sí, algo que puedan contar a sus amigos, algo que puedas contar cuando llegas a casa, que puedas decir "mira qué curioso", esto no lo había pensado, o es otra forma de verlo. Que nos sorprenda y que nos enseñe y motive"

\section{Figura 2.}

Clúster que muestra la relación entre las categorías coincidentes con el segundo objetivo.

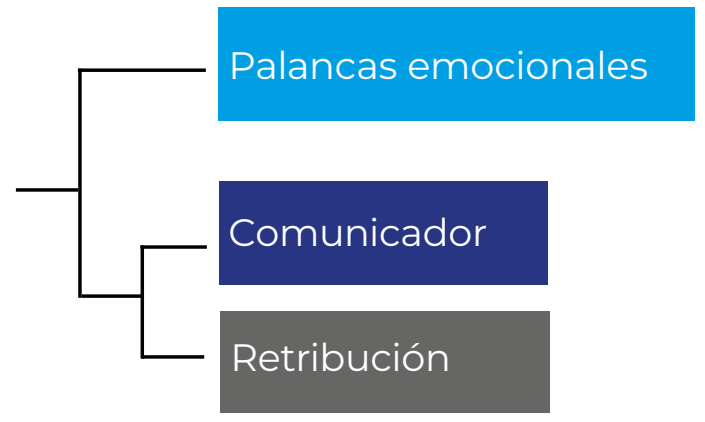

En la tabla 2 puede verse el número de referencias incluidas en cada una de las categorías, siendo la categoría "comunicador" la que presenta en porcentaje una importancia mayor, seguida de "retribución" y de "palancas emocionales". 
Tabla 2

Número de referencias incluidas en las principales categorías

\begin{tabular}{|c|c|c|c|}
\hline Categoría (nodo) & Núm. referencias & $\%$ & Núm. elementos codificados \\
\hline Comunicador & 141 & 43.3 & 12 \\
\hline Retribución & 103 & 31.5 & 12 \\
\hline Palancas emocionales & 82 & 25.2 & 12 \\
\hline Total & 326 & 100 & - \\
\hline
\end{tabular}

\section{DISCUSIÓN}

Respecto al primer objetivo, los resultados indican que el uso del storytelling como técnica es tan entrenable como cualquier otra técnica, y que habrá unos individuos que tengan mejores cualidades que otros, pero en cualquier caso con el entrenamiento mejorarán las cualidades de todos ellos. Resultado avalado por algunos autores que sostienen que a lo largo de la vida los profesionales deberían ir descubriendo el conjunto de habilidades para la comunicación pública participando de un proceso de autoaprendizaje que los lleve a desarrollar estrategias que den lugar a un estilo personal (Del Barrio y Borragán, 2005; Del Barrio y Borragán, 2011; Del Barrio y Del Barrio, 2018; Evans et al., 2004).

También respecto al primer objetivo los resultados indican que la persuasión entendida desde el punto de vista motivacional, es decir, guiar, motivar, inspirar y educar (Porter, 2008), debería ser considerada como elemento clave de la formación. El programa de entrenamiento que se diseñe debería incluir la preparación necesaria. Estudios como el de Auvinen et al. (2013) concluyen que los líderes utilizan las historias para motivar, inspirar, reducir conflictos, influir en los superiores y establecer una dirección clara. Nada de esto es ajeno al ámbito de la educación de estudiantes universitarios, quienes, bien como personas que reciben la influencia de un líder o bien como personas que lideran a otras, deben conocer qué es, cómo influye y cómo se utiliza el storytelling, ya que el liderazgo en educación es considerado como una de las competencias transversales más importantes (Cleverley-Thompson, 2018; Delors, 1996; Perrenoud, 2004; Reyzábal, 2012). En este estudio se asume 
que en el ámbito educativo la persuasión no busca manipular, sino liderar un cambio actitudinal como consecuencia de una exposición a una propuesta persuasiva (Briñol et al., 2007; Petty y Cacioppo, 1983) en donde se produce un intercambio donde ganan comunicador y audiencia (Cialdini, 2009).

Respecto al segundo objetivo, los resultados indican que las claves para el diseño de un programa que entrene en el uso del storytelling como técnica están recogidas en las categorías de: 1) "Comunicador", 2) "Retribución" y 3) "Palancas emocionales". Respecto a la primera se resumiría en "empatía" y se concreta en que el comunicador debería utilizar lenguaje sencillo y adecuado a la audiencia, conocer los intereses de la audiencia antes de dirigirse a ella, captar la tensión emocional para adaptase a ella. Además, debe disponer de conocimientos profundos del tema sobre el que habla, Respecto a la segunda los resultados indican que debería tenerse en cuenta qué es aquello que la audiencia (alumnado) espera. Esta investigación apunta que la audiencia no presta de forma gratuita su atención a alguien (tampoco un alumno al profesor), sino que quiere sentirse retribuida (Mora, 2017). Ante la cantidad de información, formación, etcétera, la audiencia se muestra cada vez más selectiva y crítica y quiere llevarse algo. Ese "algo" no es únicamente cognición o saberes, sino que tiene que ver con el crecimiento y desarrollo personal (Cortés, 2003). En este trabajo se asume, que esa capacidad de retribución que tenga un docente o un discente cuando le toque comunicar alguna cuestión, será lo que lo haga diferente del resto y lo haga imprescindible y no sustituible por las tecnologías. El comunicador y su relato son indisociables (Galar et al., 2021) en un mundo que vive en la modernidad líquida, en donde la creación de secuencias narrativas se vuelve cada vez más difícil, abandonando la cultura de aprendizaje para instalarse en la cultura del desapego, la discontinuidad y el olvido (Bauman, 2015). Así, cultivar la voz personal de un docente se vuelve una cuestión importante para poder proporcionar orientación desde el liderazgo. Respecto a la tercera, el componente subjetivo de la audiencia se ve implicado por una suma de las dos anteriores, cuando el comunicador conecta empáticamente con la audiencia y esta siente que está obteniendo lo 
que espera (o más de lo que espera) a cambio de su atención.

El análisis también ofrece algunos resultados contrarios a lo asumido en el planteamiento de la investigación y que se estima interesantes para futuras investigaciones. Uno de ellos es que la reputación del comunicador no es demasiado influyente a la hora de asumir el contenido de la comunicación. Una reputación que no se revalida en cada comunicación contribuye a lo contrario, a un deterioro de la imagen del comunicador y una pérdida de credibilidad. La reputación adquirida cada vez es menos sólida si no se mantiene. Este resultado está alineado con la investigación de Casillas et al. (2015) que sostiene que la categoría profesional del docente no lo hace mejor a la vista de sus alumnos y no la consideran importante para la calidad del desempeño docente del profesor o de la profesora.

La otra cuestión inesperada que surge de los resultados es la idea de que el storytelling puede usarse prácticamente en todos los campos salvo en uno muy concreto y aislado, el científico entre científicos, que prefieren una comunicación más cognitiva, racional y exenta de cualquier emoción. Esta limitación no solo no invalida el uso del storytelling en el resto de ámbitos, sino que sirve de contraste para ver que hay muy pocos terrenos, solo uno, en los que el storytelling no es considerado por el panel de expertos como técnica comunicativa útil. Se excluye de la esfera puramente científica la comunicación divulgativa de la ciencia que a menudo acude a metáforas y analogías para explicar lo difícilmente comprensible en lenguaje científico.

\section{CONCLUSIONES}

Comunicar de forma eficaz en el ámbito educativo es fundamental, por lo que la introducción de técnicas innovadoras (Fidalgo-Blanco, Sein-Echaluce y García-Peñalvo, 2019; García-Peñalvo, 2016) como el storytelling se revela adecuada. Esto está alineado con la idea de la European Association for Quality Assurance in Higher Education (2014) que dice que la enseñanza universitaria demanda excelencia. Y con trabajos como el de Romero et al. (2017) que concluyen la conveniencia de enfatizar la formación en competencias ligadas a las relaciones interpersonales, en 
concreto, aquellas que tienen que ver con la comunicación oral y escrita, por ser las más demandadas por los egresados una vez se incorporan al ejercicio profesional.

Por lo tanto, en relación a los objetivos fijados se concluye: 1) que sí es entrenable el uso del storytelling como técnica; 2) que las claves principales tienen que ver con las habilidades del comunicador para conectar con la audiencia a través de un relato que tenga relación con sus intereses; 3) que la audiencia quiere obtener algo a cambio de su tiempo de atención, y no solo se trata de conocimiento, también de motivación, guía, orientación.

Es importante señalar que esta investigación cuenta con algunas limitaciones, la primera y más importante es la propia metodología elegida, una investigación de carácter exploratorio caracterizada, entre otras cosas, por su carácter subjetivo y superficial al no tener antecedentes que pueda servir para orientar a la investigación (Morales, 2015). Sin embargo, resultan muy útiles en cuanto a prospectiva porque plantean nuevos interrogantes a abordar con otro tipo de metodología menos flexible, amplia o dispersa (Morales, 2015). Se estima que en este estudio se ha abierto una amplia prospectiva a futuras investigaciones con metodología y técnicas que puedan ofrecer resultados más sólidos.

La otra limitación de la investigación reside en el número de participantes, aunque según Báez (2006) número adecuado en un análisis Delphi, se estima que un número mayor hubiera dado mayor consistencia a los resultados, pero resultaba difícil encontrar participantes que reunieran la tres características (profesionales que usan storytelling en su ámbito profesional, docentes en educación formal de adultos, y con formación en storytelling).

\section{REFERENCIAS}

Álvarez, A. R. (2014). El proceso es un cuento: el storytelling en la docencia del Derecho procesal. Reeduca (Derecho), 5(1).

Auvinen, T., Aaltio, I. y Blomqvist, K. (2013). Constructing leadership by storytellingthe meaning of trust and narratives. Leadership \& Organization Development Journal, 34(6), 496-514. 
Báez, J. (2006). Investigación cualitativa. Esic Editorial.

Barthes, R., Bremond, C., Dorriots, B., Genette, G., Greimas, A. J., Gritti, J., ... Todorov, T. (1970). Análisis estructural del relato. Editorial Tiempo Contemporáneo.

Bauman, Z. (2015). Modernidad líquida. Fondo de cultura económica.

Bautista, A. (2009). Relaciones interculturales en educación mediadas por narraciones audiovisuales. Comunicar, 17(33), 149-156.

Bietti, L. M., Tilston, O. y Bangerter, A. (2019). Storytelling as adaptive collective sensemaking. Topics in cognitive science, 17(4), 710-732. https://doi.org/10.1111/ tops.12358

Briñol, P., Horcajo, F. J., Valle, C. y De Miguel, J. M. (2007). Cambio de actitudes a través de la comunicación. Psicología social, 491-516.

Cabero, J. y Infante, A. (2014). Empleo del método Delphi y su empleo en la investigación en comunicación y educación. Edutec. Revista electrónica de tecnología educativa 48, a272.

Casillas, S., Cabezas, M. y Pinto, A. M. (2015). University Professor's evaluation: Does a higher category make a better professor? Education in the knowledge society, 16(3), 106-123.

Cialdini, R. B. (2009). Influence: Science and practice (Vol. 4). Pearson education.

Cleverley-Thompson, S. (2018). Teaching storytelling as a leadership practice. Journal of Leadership Education, 17(1), 132-140. https://doi.org/10.12806/V17/11/A1

Cortés, A. (2003). Implicaciones psicopedagógicas de un desarrollo moral íntegro: la educación holística. Revista Iberoamericana de Educación, 33(2), 1-13.

De La Fuente, S. (2011). Análisis conglomerados. Facultad Ciencias Económicas y Empresariales, Universidad Autónoma de Madrid.

Del Barrio, J. y Borragán, A. (2005, febrero 7). El arte de Hablar. Prevenir los problemas de Voz. V Congreso Internacional Virtual de Educación, Madrid, España.

Del Barrio, J. A. y Borragán, A. (2011). Cómo atraer la atención hablando. Un reto para la enseñanza. Bordón. Revista de pedagogía, 63(2), 15-26. 
Del Barrio, J. A. y Del Barrio, Á. (2018). Análisis de la habilidad comunicativa docente y pautas de actuación. Atraer la atención hablando: un reto para la enseñanza universitaria. International Journal of Developmental and Educational Psychology. Revista INFAD de Psicología, 7(1), 73-84. https://doi. org/10.17060/ijodaep.2018.n1.v1.1160

Delors, J. (1996). Informe a la UNESCO de la Comisión Internacional sobre la Educación para el Siglo XXI. La educación encierra un tesoro, 34.

Díaz, L., Torruco, U., Martínez, M. y Varela, M. (2013). La entrevista, recurso flexible y dinámico. Investigación en educación médica, 2(7), 162-167.

Duveskog, M., Tedre, M., Sedano, C. I. y Sutinen, E. (2012). Life Planning by digital storytelling in a primary school in rural Tanzania. Journal of Educational Technology \& Society, 15(4).

European Association for Quality Assurance in Higher Education, E. (2014). The concept of excellence in higher education. Occasional Papers, 20.

Evans, A., Evans, V., Lami, A. y Jones, O. (2004). Public speaking in a democracy. Journal of instructional Psychology, 37(4), 325.

Ferrés, J. y Masanet, M.-J. (2017). La eficacia comunicativa en la educación: potenciando las emociones y el relato. Comunicar, 25(52), 51-60. https://doi. org/10.3916/C52-2017-05

Fidalgo-Blanco, Á., Sein-Echaluce, M. y García-Peñalvo, F. (2019). ¿Pueden las tendencias de innovación educativa predecir los cambios que transformarán el modelo educativo? https://doi.org/10.5281/zenodo.2672967

Flick, U. (2012). Introducción a la Investigación Cualitativa. Ediciones Morata.

Galar, J.L., Cortés, A. y Orejudo, S. (2021). El como técnica capaz de contribuir al aprendizaje a través del aumento del recuerdo inmediato y de la comprensión de las materias en alumnos universitarios. [Tesis doctoral, Universidad de Zaragoza]. Repositorio Institucional Unizar. https://zaguan.unizar. es/record/106302

García-Peñalvo, F. J. (2016, 21 de noviembre). En clave de innovación educativa. 
Construyendo el nuevo ecosistema de aprendizaje. I Congreso Internacional de Tendencias en Innovación Educativa, CITIE 2016. Arequipa, Perú.

Goleman, D. (2006). Emotional intelligence. Bantam.

Greene, E. (1996). : Art and Technique: ABC-CLIO.

Harari, Y. N. (2016). Homo Deus: breve historia del mañana: Debate.

Hernández, R. (2014). La investigación cualitativa a través de entrevistas: su análisis mediante la teoría fundamentada. Cuestiones Pedagógicas. Revista de Ciencias de la Educación (23), 187-210.

Hernández, R., Collado, C. y Lucio, P. (2010). Metodología de la investigación. McGraw-Hill.

López, A. P. H. y Vargas-Monardes, J. (2013). La política relatada: el Storytelling de Barack Obama en el marco de la Operación Gerónimo. Palabra clave, 16(1), $12-44$.

Mar, R. A. (2011). The neural bases of social cognition and story comprehension. Annual review of psychology, 62, 103-134.

Mellmann, K. (2012). Is storytelling a biological adaptation. Telling stories: literature and evolution. De Gruyter.

Merriam, S. y Tisdell, E. (2009). Qualitative research: A guide to design and implementation. John Wiley\& Sons, Inc.

Mora, F. (2017). Neuroeducación. Alianza Editorial.

Morales, N. (2015). Investigación exploratoria: tipos, metodología y ejemplos. https:// www.lifeder.com/investigacion-exploratoria

Paiano, A. (2019). "Storytelling": prácticas narrativas para la formación de docentes. En J.L. Rodríguez y C.Annacontini (Coords.), Metodologías narrativas en educación (pp. 169-178). Ediciones de la Universidad de Barcelona.

Patton, M. Q. (1987). How to use qualitative methods in evaluation. Sage.

Perrenoud, P. (2004). Diez nuevas competencias para enseñar. Graó.

Petty, R. E. y Cacioppo, J. T. (1983). Central and peripheral routes to persuasion: Application to advertising. Advertising and consumer psychology, 1, 3-23. 
Porter, B. (2008). Digital storytelling. San Jose, CA: Adobe Systems Incorporated. www.adobe.com/education/instruction/adsc/pdf/digital_storytelling.pdf.

Pozo, M. T., Gutierrez, J. y Rodríguez, C. (2007). El uso del método Delphi en la definición de los criterios para una formación de calidad en animación sociocultural y tiempo libre. Revista de investigación educativa, 25(2), 351-366.

Reguant, M. y Torrado, M. (2016). El método Delphi. REIRE. Revista d'Innovació i Recerca en Educació, vol. 9, (2), p. 87-102. https://doi.org/10.1344/reire2016.9.1916

Reyzábal, M. V. (2012). Las competencias comunicativas y lingüísticas, clave para la calidad educativa. REICE: Revista Iberoamericana sobre Calidad, Eficacia y Cambio en Educación, 10(4), 63-77.

Romero, M. R., Castejón, F.-J., López, V.-M. y Fraile, A. (2017). Evaluación formativa, competencias comunicativas y TIC en la formación del profesorado. Comunicar, 52(25), 73-82. https://doi.org/10.3916/C52-2017-07

Salmon, C. (2008). : la máquina de fabricar historias y formatear las mentes. Península.

Sandín, M. (2003). Investigación cualitativa en educación. Fundamentos y tradiciones. Mcgraw Hill.

Stubbersfield, J. M., Flynn, E. G. y Tehrani, J. J. (2017). Cognitive evolution and the transmission of popular narratives: a literature review and application to urban legends. Evolutionary Studies in Imaginative Culture, 1(1), 121-136.

Sugiyama, M. S. (2001). Food, foragers, and folklore: The role of narrative in human subsistence. Evolution and Human Behavior, 22(4), 221-240.

Tolisano, S. R. (2009). Digital tools for educators.

Vázquez, P. (2016). El «storytelling» en la literatura científica española: hacia un estado de la cuestión. adComunica, 12, 27-44. https://doi.org/10.6035/21740992.2016 .12 .3

Villardón, J. L. V. (2007). Introducción al análisis de clúster. Departamento de Estadística, Universidad de Salamanca. https://www.coursehero.com/ 
file/65735977/AnalisisCluster5pdf/

Webler, T., Levine, D., Rakel, H. y Renn, O. (1991). A novel approach to reducing uncertainty: the group Delphi. Technological forecasting and social change, 39(3), 253-263.

Yin, R. K. (2015). Qualitative research from start to finish. Guilford Publications.

Fecha de recepción 18 de mayo de 2021

\section{Fecha de aceptación 15 de septiembre de 2021}

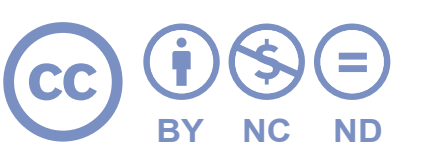

Este artículo pertenece a la Universidad de Zaragoza y se distribuye bajo una Licencia Creative Commons Atribución-NoComercial-SinDerivar 4.0 Internacional.

Eres libre de compartir copiar y redistribuir el material en cualquier medio o formato -

Bajo las condiciones siguientes:

- Reconocimiento de la autoría, ya incluida en esta diapositiva.

- NoComercial - no se puede utilizar el material para una finalidad comercial.

- SinObraDerivada - Sin remezclar, transformar o crear a partir del material. 